CERN-TH/95-31

GeF-TH-2/95

\title{
SCALE DEPENDENCE AND SMALL $x$ BEHAVIOUR OF POLARIZED PARTON DISTRIBUTIONS
}

\author{
Richard D. Ball*, Stefano Forte $\dagger$ and Giovanni Ridolfi \\ Theory Division, CERN, \\ CH-1211 Genève 23, Switzerland.
}

\begin{abstract}
We discuss perturbative evolution of the polarized structure function $g_{1}$ in the $\left(x, Q^{2}\right)$ plane, with special regard to the small- $x$ region. We determine $g_{1}$ in terms of polarized quark and gluon distributions using coefficient functions to order $\alpha_{s}$. At small $x g_{1}$ then displays substantial scale dependence, which necessarily implies a corresponding scale dependence in the large $x$ region. This scale dependence has significant consequences for the extraction of the first moment from the experimental data, reducing its value while increasing the error. Conversely, the scale dependence may be used to constrain the size of the polarized gluon distribution.
\end{abstract}

CERN-TH/95-31

February 1995

* On leave from a Royal Society University Research Fellowship.

$\dagger$ On leave from INFN, Sezione di Torino, Italy.

$\ddagger$ On leave from INFN, Sezione di Genova, Italy. 


\section{Introduction}

Experimental data on the polarized proton structure function $g_{1}$ 回 have substantially improved within the last few months [2,3], and results of comparable accuracy for the deuteron should be available soon [4]. Not only are statistical errors now substantially reduced [3], but also data which extend to rather small values of $x$ (of order 0.003 [2]) are available, as well as determinations of the structure function at different scales for a given value of $x$. This suggests that it may be possible to see the effects of perturbative QCD evolution in the data. Indeed, the contribution of the polarized gluon distribution to $g_{1}$ which appears in next-to-leading order, has been put forward [5] as one possible way of understanding the observed smallness of the first moment

$$
\Gamma_{1}\left(Q^{2}\right) \equiv \int_{0}^{1} g_{1}\left(x, Q^{2}\right) d x
$$

it may now be possible to test for such a contribution by examining the $x$ and $Q^{2}$ dependence of the structure function data. Also, the small- $x$ data [2] indicate (albeit with large experimental uncertainty) a rise of the structure function at small $x$. Such a rise, even if not present in $g_{1}$ at low scales, is generated at higher scales through perturbative QCD evolution [6] by a similar mechanism to that which drives the rise in the unpolarized structure function $F_{2}^{P}$ at small $x$ [7] recently observed at HERA [8].

A quantitative understanding of the scale dependence and the small- $x$ behaviour of the structure function is required in order to extract accurately its moments from the data: because data are taken at different values of $Q^{2}$ for each $x$ bin, and over a limited range in $x$, it is necessary to evolve them to a common scale $t=\ln \left(Q^{2} / \Lambda^{2}\right)$ and extrapolate them to all $0 \leq x \leq 1$ in order to determine any moment of $g_{1}$. The evolution is usually performed by the experimental collaborations [2,3] by assuming [9] that the virtual photon scattering asymmetry]

$$
A_{1}\left(x, Q^{2}\right) \equiv \frac{\sigma_{1 / 2}-\sigma_{3 / 2}}{\sigma_{1 / 2}+\sigma_{3 / 2}}=g_{1}\left(x, Q^{2}\right) \frac{2 x\left[1+R\left(x, Q^{2}\right)\right]}{F_{2}\left(x, Q^{2}\right)}
$$

is scale independent, so that the scale dependence of $g_{1}$ is given by that of the unpolarized structure function $F_{2}$, which is known rather accurately. This can however be a rather poor approximation: as we shall see below, at small $x \frac{d \ln A_{1}}{d t}$ diverges, and thus a large

1 We follow the notations and conventions of ref. [1], to which the reader is referred for theoretical background. 
error can be made if it is assumed that $A_{1}$ is scale independent in that region. Likewise, the small- $x$ extrapolation is usually made by assuming Regge behaviour, which is not necessarily preserved by perturbative QCD evolution. This is especially dangerous since the contribution of the unmeasured small- $x$ region to the first moment $\Gamma_{1}$ eq. (1.1) is extrapolated from the lowest $x$ data, which are necessarily taken at rather low values of $Q^{2}$. The small- $x$ contribution accounts for about $5 \%$ of the first moment [3], and because of large cancellations can give some 20 or $30 \%$ of the physically interesting singlet component of $\Gamma_{1}[\mathbb{1}]$.

A detailed investigation of the expected $x$ and $Q^{2}$ dependence of $g_{1}$, especially at small $x$, is thus called for. A first study of the scale dependence of the asymmetry eq. (1.2) was performed in ref. [10], where the one-loop Altarelli-Parisi evolution equations were linearized in $\ln t$ to determine the approximate correction to be applied to the asymmetry data. The corresponding correction to the measured first moment turns out to be very small (of the order of a few percent, i.e. smaller than statistical uncertainties). However, the effect on individual data points may be appreciable; also, since these corrections are correlated, relatively small effects on single data points can lead to a significant change of the integral computed over a finite region of $x$. Of course, these effects would approximately cancel out if the integral could be measured over the whole range $0 \leq x \leq 1$ (due to the one-loop conservation of the first moment of $\left.g_{1}\right)$. Such scale dependence corrections are thus increasingly significant as both the statistical quality and coverage of the small- $x$ region of the data improves. It is then important to investigate the effects of the nextto-leading order coupling of polarized gluons to $g_{1}$, in particular at small $x$, where the polarized gluon distribution will diverge.

In the present paper we will analyze the $x$ and $Q^{2}$ dependence of $g_{1}$, including all the available theoretical [11] and experimental [2,3] information. In particular, we will study the effects of including the coupling (which only appears at two loops) of the polarized gluon distribution to $g_{1}$, and discuss the perturbative QCD prediction for the behaviour of $g_{1}$ at small $x$. Even though a fully consistent two-loop determination of $g_{1}$ is not yet possible due to the lack of knowledge of the full set of two-loop Altarelli-Parisi splitting functions, we will show that an approximate treatment suggests that evolution effects are significantly enhanced by the gluon contribution to $g_{1}$, and that the inclusion of this contribution is necessary to understand current data. The size of these effects is essentially controlled by the size of the polarized gluon distribution; because this is unknown, it introduces an uncertainty in the determination of $g_{1}$. However, especially in the small $x$ region, 
interference between the perturbative evolution of gluon and quark contributions may lead to large fluctuations of $g_{1}$, so that the data may be used to constrain the size of this gluon contribution. We will discuss how present and future data can be used to determine the nature of the small- $x$ behaviour of polarized parton distributions, thereby extracting information on the polarized gluon distribution. Also, we will discuss the impact of these evolution effects on the extractions from the data of the first moment of $\Gamma_{1}$, and show it to be not negligible at the level of accuracy of current experiments.

\section{Scale Dependence}

Our starting point is the relation between the polarized structure function $g_{1}$ and the polarized quark and gluon distributions $\Delta q_{i}$ and $\Delta g$, which in general reads [11,12]

$$
\begin{aligned}
g_{1}(x, t)=\frac{\left\langle e^{2}\right\rangle}{2} \int_{x}^{1} \frac{d y}{y}\left\{C_{q}^{\mathrm{S}}\left(\frac{x}{y}, \alpha_{s}(t)\right) \Delta \Sigma(y, t)\right. & +C_{q}^{\mathrm{NS}}\left(\frac{x}{y}, \alpha_{s}(t)\right) \Delta q^{\mathrm{NS}}(y, t) \\
& \left.+2 n_{f} C_{g}\left(\frac{x}{y}, \alpha_{s}(t)\right) \Delta g(y, t)\right\}
\end{aligned}
$$

where $\Delta \Sigma$ and $\Delta q^{\mathrm{NS}}$ are respectively the singlet and nonsinglet quark distributions

$\Delta \Sigma(x, t)=\sum_{i=1}^{n_{f}}\left(\Delta q_{i}(x, t)+\Delta \bar{q}_{i}(x, t)\right), \quad \Delta q^{\mathrm{NS}}(x, t)=\sum_{i=1}^{n_{f}} \frac{e_{i}^{2}-\left\langle e^{2}\right\rangle}{\left\langle e^{2}\right\rangle}\left(\Delta q_{i}(x, t)+\Delta \bar{q}_{i}(x, t)\right)$,

and $n_{f}$ is the number of active flavours, with electric charge $e_{i},\left\langle e^{2}\right\rangle=\sum e_{i}^{2} / n_{f}$. The perturbative part of the $x$ and $t$ dependence of the polarized quark and gluon distributions is given by the Altarelli-Parisi equations [13]: the singlet quark and the gluon mix according to

$$
\begin{aligned}
& \frac{d}{d t} \Delta \Sigma(x, t)=\frac{\alpha_{s}(t)}{2 \pi} \int_{x}^{1} \frac{d y}{y}\left[P_{q q}^{\mathrm{S}}\left(\frac{x}{y}, \alpha_{s}(t)\right) \Delta \Sigma(y, t)+2 n_{f} P_{q g}\left(\frac{x}{y}, \alpha_{s}(t)\right) \Delta g(y, t)\right] \\
& \frac{d}{d t} \Delta g(x, t)=\frac{\alpha_{s}(t)}{2 \pi} \int_{x}^{1} \frac{d y}{y}\left[P_{g q}\left(\frac{x}{y}, \alpha_{s}(t)\right) \Delta \Sigma(y, t)+P_{g g}\left(\frac{x}{y}, \alpha_{s}(t)\right) \Delta g(y, t)\right]
\end{aligned}
$$

while the nonsinglet quark evolves independently as

$$
\frac{d}{d t} \Delta q^{\mathrm{NS}}(x, t)=\frac{\alpha_{s}(t)}{2 \pi} \int_{x}^{1} \frac{d y}{y} P_{q q}^{\mathrm{NS}}\left(\frac{x}{y}, \alpha_{s}(t)\right) \Delta q^{\mathrm{NS}}(y, t)
$$

In eqs. (2.1)-(2.2) we have taken both the factorization scale and the renormalization scale equal to $Q^{2}$, so that all scale dependence appears through $t=\ln \frac{Q^{2}}{\Lambda^{2}}$. A different choice of factorization scale is discussed in ref. [11]. 
The coefficient functions which relate the polarized parton distributions to $g_{1}$ according to eq. (2.1) are given in the leading logarithmic approximation (used in ref. [10]) by

$$
C_{q}^{(0), \mathrm{S}}\left(x, \alpha_{s}\right)=C_{q}^{(0), \mathrm{NS}}\left(x, \alpha_{s}\right)=\delta(1-x), \quad C_{g}\left(x, \alpha_{s}\right)=0,
$$

so that $g_{1}$ is just a linear combination of polarized quark distributions, whose $Q^{2}$ dependence is entirely specified by eqs. (2.3)-(2.4) (with the leading order splitting functions calculated in ref. [13]).

Beyond leading order, splitting functions and coefficient functions are no longer universal, hence even though the scale dependence of the (observable) structure function $g_{1}$ is determined uniquely, at least up to higher order corrections, its separation into contributions due to quarks and gluons is scheme dependent and thus essentially arbitrary. This ambiguity is somewhat constrained in the polarized case because of the anomalous conservation law satisfied by the singlet axial current [14], whose nucleon matrix element is proportional to the singlet component of the first moment $\Gamma_{1}$ eq. (1.1) of $g_{1}$ [1]. The anomaly has the important implication [5] that the gluon contribution to $g_{1}$, although formally of order $\alpha_{s}$, does not decouple as $Q^{2} \rightarrow \infty$. We thus expect the leading order approximation to $g_{1}$ to be particularly poor in this case.

Unfortunately, while all the coefficient functions have been calculated up to order $\alpha_{s}^{2}$ in ref. [11], only the $O\left(\alpha_{s}\right)$ corrections to the splitting functions $P^{q g}$ and $P^{q q}$ are known at present. Therefore we cannot yet perform a fully consistent next-to-leading order analysis. We will thus solve the Altarelli-Parisi eqs. (2.3) (2.4) at one loop, but include coefficient functions through order $\alpha_{s}$. The gluon coefficient function would be specified uniquely only once the next-to-leading contributions to the splitting functions are known in a particular scheme. We can partially fix it by insisting that the first moment of the polarized quark distribution be conserved by perturbative evolution. The first moment of the gluon coefficient function is then fixed by the anomaly equation to be [1]

$$
\int_{0}^{1} C_{g}\left(x, \alpha_{s}\right) d x=-\frac{\alpha_{s}}{4 \pi} .
$$

With this choice, the gluon density coincides with that which can be measured in independent hard processes [15], in that all soft contributions to $g_{1}$ are absorbed in the parton distributions and do not contribute to coefficient functions [16]. Furthermore, the first moment of $\Delta \Sigma$ coincides then with the nucleon matrix element of the canonical quark helicity operator [17]. We will further choose the higher moments of $C_{g}$ to be equal to 
the first [18]. We may then test the sensitivity of our results to the inclusion of higher order corrections by choosing alternative forms of the gluon coefficient function which still satisfy eq. (2.6), but result from different regularizations of infrared singularities [5, 16]. In this respect our approach is the same as that adopted in ref. [19]. As we shall see, evidence from the data supports the expectation that including the gluon coupling to $g_{1}$ by enforcing eq. (2.6) improves the quality of the leading order calculation, even though of course only a consistent two loop treatment will allow a definite conclusion. The inclusion of $O\left(\alpha_{s}\right)$ corrections to the quark coefficient functions is known [20,1] to have a significant effect on the determination of $\Gamma_{1}$ eq. (1.1).

With this choice, the Mellin transforms of the coefficient functions which we will use to determine $g_{1}$, defined according to $C\left(N, \alpha_{s}\right)=\int_{0}^{1} d x x^{N-1} C\left(x, \alpha_{s}\right)$ are

$$
\begin{gathered}
C_{q}^{\mathrm{NS}}\left(N, \alpha_{s}\right)=1+\frac{\alpha_{s}}{4 \pi} C_{F}\{(\psi(N)+\gamma)[3+2(\psi(N+2)+\gamma)] \\
\left.+2\left[\psi^{\prime}(N)-\frac{\pi^{2}}{6}\right]-9+\frac{6}{N}\right\}+O\left(\alpha_{s}^{2}\right) \\
C_{q}^{\mathrm{S}}\left(N, \alpha_{s}\right)=C_{q}^{\mathrm{NS}}\left(N, \alpha_{s}\right)+O\left(\alpha_{s}^{2}\right) \\
C_{g}\left(N, \alpha_{s}\right)=-\frac{\alpha_{s}}{4 \pi}+O\left(\alpha_{s}^{2}\right)
\end{gathered}
$$

where $\psi(N)=d \log \Gamma(N) / d N$. The explicit relation between the matrix element of the current and the first moments of the quark and gluon distributions is then

$$
\begin{aligned}
& \left\langle p, s\left|j_{5}^{\mu}\right| p, s\right\rangle=M s^{\mu} a_{0}\left(Q^{2}\right), \\
& a_{0}\left(Q^{2}\right)=\int_{0}^{1} d x\left[\Delta \Sigma\left(x, Q^{2}\right)-n_{f} \frac{\alpha_{s}}{2 \pi} \Delta g\left(x, Q^{2}\right)\right]
\end{aligned}
$$

where $p, M$ and $s$ are the momentum, mass and spin of the nucleon. The (scale dependent) coefficient of proportionality between $a_{0}$ and the singlet component of $\Gamma_{1}$ can be determined in terms of the first moments of the quark and gluon coefficient functions comparing eq. (2.8) with eq. (2.1).

The anomalous dimensions analogously obtained by Mellin transform of the Altarelli- 
Parisi splitting functions in eq. (2.3) are

$$
\begin{aligned}
& \gamma_{N}^{q q}=C_{F}\left[\frac{3}{2}+\frac{1}{N(N+1)}-2(\psi(N+1)+\gamma)\right]+O\left(\alpha_{s}\right) \\
& \gamma_{N}^{q g}=T_{F} \frac{N-1}{N(N+1)}+O\left(\alpha_{s}\right) \\
& \gamma_{N}^{g q}=C_{F} \frac{N+2}{N(N+1)}+O\left(\alpha_{s}\right) \\
& \gamma_{N}^{g g}=C_{A}\left[\frac{11}{6}-\frac{n_{f}}{9}+\frac{4}{N(N+1)}-2(\psi(N+1)+\gamma)\right]+O\left(\alpha_{s}\right),
\end{aligned}
$$

where, for $\mathrm{SU}(3)$ color, $C_{F}=\frac{4}{3}, C_{A}=3$ and $T_{F}=\frac{1}{2}$.

\section{Small- $x$ Behaviour}

We can now discuss the QCD predictions for the small- $x$ behaviour of $\Delta q, \Delta g$, and thus $g_{1}$. The treatment closely follows that of the unpolarized case [8]. At small $x$, the Altarelli-Parisi evolution is dominated by the lowest moments of the splitting function [7]; since these are singular at small enough $N$ the leading behaviour is found by expanding the anomalous dimensions eq. (2.9) in powers of $N$ around the rightmost singularity [21]:

$$
\begin{aligned}
& \gamma_{N}^{q q}=C_{F}\left[\frac{1}{N}+\frac{1}{2}+O(N)\right]+O\left(\alpha_{s}\right) \\
& \gamma_{N}^{q g}=T_{F}\left[-\frac{1}{N}+2+O(N)\right]+O\left(\alpha_{s}\right) \\
& \gamma_{N}^{g q}=C_{F}\left[\frac{2}{N}-1+O(N)\right]+O\left(\alpha_{s}\right) \\
& \gamma_{N}^{g g}=C_{A}\left[\frac{4}{N}-\left(\frac{n_{f}}{9}+\frac{13}{6}\right)+O(N)\right]+O\left(\alpha_{s}\right) .
\end{aligned}
$$

Following the procedure described in ref. [8], i.e. diagonalizing the matrix of anomalous dimensions, inverting the Mellin transform, substituting the resulting splitting functions into the evolution equation (2.3), and differentiating with respect to $x$, the eigenvectors $v^{ \pm}=\left(\Delta q^{ \pm}, \Delta g^{ \pm}\right)$are found to evolve in the $(x, t)$ plane according to the pair of wave-like equations

$$
\frac{\partial^{2}}{\partial \xi \partial \zeta} v^{ \pm}(\xi, \zeta)+\delta_{ \pm} \frac{\partial}{\partial \xi} v^{ \pm}(\xi, \zeta)=\gamma_{ \pm}^{2} v^{ \pm}(\xi, \zeta)
$$

The relevant variables are

$$
\xi=\ln \frac{x_{0}}{x}, \quad \zeta=\ln \frac{t}{t_{0}},
$$


and $x_{0}$ and $t_{0} \equiv \ln \left(Q_{0}^{2} / \Lambda^{2}\right)$ are arbitrary reference values of $x$ and $t$, chosen in such a way that the approximate form of the splitting functions eq.(3.1) is applicable for $x \lesssim x_{0}$ and $t \gtrsim t_{0}$. The coefficients $\gamma_{ \pm}$and $\delta_{ \pm}$are determined by the diagonalization of the singlet anomalous dimension matrix (3.1): the eigenvalues are $\lambda_{ \pm}=\frac{1}{2} \beta_{0}\left(\gamma_{ \pm}^{2} / N+\delta^{ \pm}+O(N)\right)$, with

$$
\begin{aligned}
\gamma_{ \pm}^{2} & =\frac{8}{33-2 n_{f}}\left(5 \pm 4 \sqrt{1-\frac{3 n_{f}}{32}}\right) \\
\delta_{ \pm} & =\frac{35+2 n_{f} \pm 43\left(\frac{1-11 n_{f} / 86}{\sqrt{1-3 n_{f} / 32}}\right)}{2\left(33-2 n_{f}\right),}
\end{aligned}
$$

and $\beta_{0} \equiv 11-\frac{2}{3} n_{f}$ is the leading coefficient of the QCD beta function. The eigenvectors $v^{ \pm}=\left(\Delta q^{ \pm}, \Delta g^{ \pm}\right)$are given (to leading order in $N$ ) by

$$
\Delta q^{ \pm}=-2\left(1 \mp \sqrt{1-\frac{3 n_{f}}{32}}\right) \Delta g^{ \pm}
$$

Notice that the eigenvectors are not orthogonal.

The small- $x$ evolution equations satisfied by $v^{ \pm}$are thus of exactly the same form as those satisfied by $G\left(x, Q^{2}\right)=x g\left(x, Q^{2}\right)$, where $g$ is the unpolarized gluon distribution [8]. There are two basic differences between the polarized and the unpolarized case. Firstly, since the polarized anomalous dimensions have their rightmost singularity at $N=0$, while the unpolarized ones have it at $N=1$, parton distributions here play the same role as parton distributions multiplied by $x$ in the unpolarized case. Furthermore, since in the polarized case each entry in the matrix of anomalous dimensions has a simple pole at $N=0$, the polarized quark and gluon distributions evolve together on the same footing and the eigenvector of evolution is a linear combination of them, while in the unpolarized case, since only $\gamma^{g q}$ and $\gamma^{g g}$ are singular, the gluon distribution evolves independently according to a wave equation of the form (3.2), and the quark is then determined by it.

These differences apart, the unpolarized and polarized cases have much in common since the form of the equation(3.2) is the same. In particular:

(i) The equations (3.2) are linear and causal. Their solution at $\left(x^{\prime}, t^{\prime}\right)$ is determined by the knowledge of boundary conditions given on the $x=x_{0}$ and $t=t_{0}$ axes for all $x^{\prime} \leq x \leq x_{0}$ and $t^{\prime} \geq t \geq t_{0}$.

(ii) The equations are symmetric in $\xi$ and $\zeta$, modulo the damping term proportional to $\delta_{ \pm}$. Their solutions will display this symmetry, unless $\xi \ll \zeta$, in which case the damping 
term is increasingly important because the small- $N$ expansion eq. (2.9) is beginning to break down; any further asymmetry will be due to the boundary conditions.

(iii) The general solution can be determined exactly in terms of the Green's function $I_{0}\left(2 \gamma_{ \pm} \sqrt{\xi \zeta}\right)$ of the wave operator:

$$
\begin{aligned}
v^{ \pm}(\xi, \zeta)=I_{0} & \left(2 \gamma_{ \pm} \sqrt{\xi \zeta}\right) e^{-\delta_{ \pm} \zeta} v^{ \pm}(0,0)+\int_{0}^{\xi} d \xi^{\prime} I_{0}\left(2 \gamma_{ \pm} \sqrt{\left(\xi-\xi^{\prime}\right) \zeta}\right) e^{-\delta_{ \pm} \zeta} \frac{\partial}{\partial \xi^{\prime}} v^{ \pm}\left(\xi^{\prime}, 0\right) \\
& +\int_{0}^{\zeta} d \zeta^{\prime} I_{0}\left(2 \gamma_{ \pm} \sqrt{\xi\left(\zeta-\zeta^{\prime}\right)}\right) e^{\delta_{ \pm}\left(\zeta^{\prime}-\zeta\right)}\left(\frac{\partial}{\partial \zeta^{\prime}} v^{ \pm}\left(0, \zeta^{\prime}\right)+\delta_{ \pm} v^{ \pm}\left(0, \zeta^{\prime}\right)\right)
\end{aligned}
$$

(iv) The asymptotic form of the solutions fall into two classes, according to the behaviour of the boundary conditions. Let us consider for definiteness the boundary conditions along the $\zeta=0$ axis, i.e., as a function of $x$ at fixed $t=t_{0}$. For boundary conditions on both $\Delta q$ and $\Delta g$ which are less singular than any power of $x$, the asymptotic behaviour for large $\xi$ and $\zeta$ will be universal and given by the asymptotic form of the Bessel function $I_{0}$ :

$$
v^{ \pm}(\xi, \zeta) \sim \frac{1}{\sqrt{4 \pi \gamma_{ \pm} \sqrt{\xi \zeta}}} \exp \left\{2 \gamma_{ \pm} \sqrt{\xi \zeta}-\delta_{ \pm} \zeta\right\}
$$

This behaviour holds as $\xi \zeta \rightarrow \infty$ along any curve such that $\frac{\xi}{\zeta} \rightarrow \infty$. If instead the boundary conditions have a power-like singularity of the form $x^{-\lambda}$, the asymptotic result eq. (3.7) only applies to the region $\xi<\zeta \frac{\gamma}{\lambda}$, whereas for larger values of $\xi$ we have asymptotically

$$
v^{ \pm}(\xi, \zeta) \sim \exp \left\{\lambda \xi+\left(\frac{\gamma_{ \pm}^{2}}{\lambda}-\delta_{ \pm}\right) \zeta\right\}
$$

i.e., the leading asymptotic behaviour reproduces the boundary condition. If the boundary conditions on $\Delta q$ and $\Delta g$ are different, but one (or both) has a power-like singularity, the most singular behaviour will always dominate asymptotically because of the mixing introduced by the evolution.

The properties of the small- $x$ solution to the evolution equations allow us to make some qualitative predictions on the expected behaviour of $g_{1}$ at small $x$. First, $\left|g_{1}\right|$ is expected generically to rise at small $x$ [6], at least as fast as eq. (3.7), i.e. stronger than any power of $\ln \frac{1}{x}$. This suggests that any rise of the boundary condition softer than this will soon be masked by the perturbative rise and thus not directly observable. Although both $\gamma_{+}$and $\gamma_{-}$are positive, $\gamma_{+}$is larger so asymptotically the eigenvector $v^{+}$eq. (3.5) will dominate, 
and, at small $x, \Delta q$ and $\Delta g$ will eventually have the opposite sign. The sign of the rise of $g_{1}$ is however not a priori determined, but rather is sensitive to the relative importance of the quark and gluon polarizations on the boundaries: if $\Delta g$ is sufficiently large and positive, then asymptotically $g_{1}$ will tend to minus infinity. The onset of the asymptotic behaviour will lead, for some boundary conditions, to interesting interference effects. The shape of the rise will be determined by the qualitative form of the boundary conditions: it will take the universal form eq. (3.7) for sufficiently soft boundary conditions, while it will tend to reproduce the rise in the boundary condition itself for harder ones. This is displayed in fig. 1, where we show a contour plot of the leading small- $x$ form of $g_{1}$ computed by assuming that the Altarelli-Parisi equations reduce to their small- $x$ form eq. (3.2), with "soft" (fig. 1a) and "hard" (fig. 1b) boundary conditions. If the boundary conditions are soft it is also possible to determine the asymptotic behaviour of the experimentally measured asymmetry eq. (11.2): given that the unpolarized structure function measured at HERA already displays the corresponding universal asymptotic form [8], we have

$$
A_{1}\left(x, Q^{2}\right) \sim x \exp \left[(\sqrt{2}-1) 2 \gamma \sqrt{\xi \zeta}-\frac{2}{11} \delta \zeta\right]
$$

where $\gamma$ and $\delta$ are as given in ref. [8], and we have neglected some very small $n_{f}$ dependent corrections. Thus, even though at small $x$ the asymmetry falls as $x, \ln \left(\frac{1}{x} A_{1}\right)$ grows linearly with $\sqrt{\ln (1 / x) \ln t}$.

Putting everything together, we expect that if sufficiently numerous and precise data on $g_{1}$ at small $x$ and large $Q^{2}$ are available (say, $x<0.1$ and $Q^{2}>10 \mathrm{GeV}^{2}$ ) then it should be possible, just as in the unpolarized case [8], to determine experimentally whether the small- $x$ structure function and parton distributions are soft or hard. Clearly, this is not yet the case, since only a couple of the SMC data points [2] and none of the E143 data [3] fall within this range. This is demonstrated explicitly in fig. 1, where a scatter plot of the small $x$ data is superimposed on the leading predicted asymptotic small $x$ behaviour for soft and hard boundary conditions: it is clear that the data are insufficient to distinguish between the two scenarios simply because only a few of the experimental points fall close to the asymptotic small- $x$ region.

We expect however that even with a few data at small $x$ and reasonable $Q^{2}$ it should be possible to constrain the size of the gluon polarization, given the very strong sensitivity of $g_{1}$ and its scale dependence to it. Finally, because the small $x$ eigenvector is a linear combination of quark and gluon (with a large coefficient multiplying the gluon contribution), and because both the quark and the gluon distributions grow at small $x$ but with 
opposite signs, we expect in general the appearance in $g_{1}$ of fluctuations due to interference between quark and gluon contributions, until the asymptotic behaviour sets in. The observation of such fluctuations would put a strong constraint on the shape and size of the gluon, although presumably now in a non-universal way (i.e., dependent on the particular form of the boundary conditions).

\section{The Data}

In order to establish some more quantitative results, we need direct input from the experimental data. We use the SMC and E143 determinations of the scattering asymmetry eq. (1.2) [2, 3], together with the NMC determination of the unpolarized structure function $F_{2}$ [22 and the SLAC determination [23] of the ratio $R\left(x, Q^{2}\right)$ of the longitudinal to transverse virtual photoabsorption cross section, to obtain values for $g_{1}\left(x, Q^{2}\right)$ according to eq. (1.2) 2. This provides us with a determination of $g_{1}$ along a set of points $\left(x_{k}, Q_{k}^{2}\right)$ in the $\left(x, Q^{2}\right)$ plane; due to the kinematic coverage of the present experiments these points lie for each experiment on a curve which is a monotonically rising function $Q_{\exp }^{2}(x)$. We then choose a physically motivated parametrization of the initial polarized quark and gluon distributions $\Delta q_{i}$ and $\Delta g$ as a function of $x$ for all $0 \leq x \leq 1$ at a reference starting scale $Q^{2}=Q_{0}^{2} ; g_{1}$ is then determined for all $Q^{2} \geq Q_{0}^{2}$, and in particular at each data point $\left(x_{k}, Q_{k}^{2}\right)$, by means of eq. (2.1) and the evolution equations (2.3), 2.4). The free parameters in the initial parametrization are determined by a best fit of the evolved distribution to all the available data. Notice that, because the Altarelli-Parisi evolution is causal, the structure function determined in this way for all points $\left(x, Q^{2}\right)$ such that $Q^{2}>Q_{\exp }^{2}(x)$ is independent of the specific parametrization, to the extent that the data determine the shape of $g_{1}$ along the line $Q_{\exp }^{2}(x)$. The only real model dependence here comes in the way $g_{1}$ is decomposed into quark singlet and nonsinglet and gluon contributions.

We parametrize the initial quark and gluon distributions as

$$
\begin{aligned}
\Delta \Sigma\left(x, Q_{0}^{2}\right) & =N\left(\alpha_{q}, \beta_{q}, a_{q}\right) \eta_{q} x^{\alpha_{q}}(1-x)^{\beta_{q}}\left(1+a_{q} x\right) \\
\Delta q^{\mathrm{NS}}\left(x, Q_{0}^{2}\right) & =N\left(\alpha_{\mathrm{NS}}, \beta_{\mathrm{NS}}, a_{\mathrm{NS}}\right) \eta_{\mathrm{NS}} x^{\alpha_{\mathrm{NS}}}(1-x)^{\beta_{\mathrm{NS}}}\left(1+a_{\mathrm{NS}} x\right) \\
\Delta g\left(x, Q_{0}^{2}\right) & =N\left(\alpha_{g}, \beta_{g}, a_{g}\right) \eta_{g} x^{\alpha_{g}}(1-x)^{\beta_{g}}\left(1+a_{g} x\right),
\end{aligned}
$$

2 For consistency we do not include kinematic higher twist corrections in determining $g_{1}$ from $A_{1}$, as was done in ref. [3], since these are not included in the extraction [22] of $F_{2}$ from the data, and because there exists no systematic treatment of higher twist corrections to either $g_{1}$ or $F_{2}$. 
where $N(\alpha, \beta, a)$ is fixed by the normalization condition

$$
N(\alpha, \beta, a) \int_{0}^{1} d x x^{\alpha}(1-x)^{\beta}(1+a x)=1 .
$$

Similar parametrizations have been used in recent global fits of polarized parton distributions [19]. The large $x$ behaviour of the parametrization eq. (4.1) is inspired by QCD counting rules [24], from which we expect $\beta_{q}=\beta_{\mathrm{NS}} \simeq 3$ and $\beta_{g} \simeq 4$. The polynomial in $x$ (which we take to be first order) is a phenomenological interpolation which has the purpose of improving the quality of the fit in the intermediate $x$ region.

Several estimates for the small $x$ behaviour of $g_{1}$ are available. Arguments based on the dominance of known Regge poles [25] lead to the expectation

$$
g_{1} \underset{x \rightarrow 0}{\sim} x^{\lambda} ; \quad 0 \leq \lambda \leq 0.5
$$

However, coherence arguments [24] suggest that (at a typical nucleon scale) the polarized gluon distribution should be related to the unpolarized one $g(x)$ according to

$$
\frac{\Delta g}{g} \underset{x \rightarrow 0}{\sim} x
$$

Thus, if the behaviour of is $g$ dominated by a soft pomeron [26], then $g(x) \sim \frac{1}{x}$ so that the lower bound for $\lambda$ in eq. (4.3) is saturated, but if $g(x)$ has a harder behaviour then $\lambda<0$ ( $\lambda \sim-0.1$ with a supercritical pomeron, and even lower with the Lipatov hard pomeron [26], however values as low as -0.5 are now clearly ruled out by data from HERA [8]). A model of the pomeron based on nonperturbative gluon exchange [27] gives the still singular but softer behaviour

$$
g_{1} \underset{x \rightarrow 0}{\sim}-2 \ln x
$$

Finally, it has been argued [28] that it is possible for negative signature cuts to induce an extremely singular behaviour

$$
g_{1} \underset{x \rightarrow 0}{\sim} \frac{1}{x \ln ^{2} x}
$$

Nonperturbative arguments do not generally distinguish between the leading behaviour of the quark and that of the gluon. We will hence consider several representative cases of soft or hard boundary conditions of the form eq. (4.1) for both the quark and 
gluon distributions.3 Notice, however, that since QCD evolution mixes the quark and gluon distributions (as in eq. (3.5)), if either of the two has a very singular behaviour, on propagation the stronger singularity will dominate both. Also, behaviours of the form of eq. (4.5) or eq. (4.3) with $\lambda \lesssim 0.3$ will grow rapidly upon evolution according to eq. (3.7) and thus their precise form will be hidden by the perturbatively generated rise of $g_{1}$.

We choose the starting scale at which the parametrization eq. (4.1) is imposed to be $Q_{0}^{2}=1 \mathrm{GeV}^{2}$. Here, this has the advantage that $Q_{0}^{2}<Q_{\exp }^{2}(x)$ for all $x$, so that we may compare to the data without having to evolve $g_{1}$ backwards in $Q^{2}$ (which would in general be unstable, due to the causal nature of the Altarelli-Parisi equations). Of course the price to be paid for this choice is that at such a low scale there could be sizable higher twist effects. However, in the unpolarized case these effects seem mostly to be concentrated in the large $x$ region [29], where $Q_{\exp }^{2}(x)$ is large. Because the evolution of the leading twist is decoupled from that of the higher twist contributions, the overall effect on our results of having neglected higher twist corrections should be small. However, our final results for $g_{1}\left(x, Q^{2}\right)$ should only be trusted in the region where the higher twist effects are small, and in particular do not necessarily provide an accurate description of the data at large $x$ $(x \gtrsim 0.3)$ and small scales $\left(Q^{2} \sim Q_{0}^{2}\right)$.

At $Q^{2}=Q_{0}^{2}$, the quark distributions should be dominated by the valence component, hence we will take $\beta_{q}=\beta_{\mathrm{NS}}$ and $a_{q}=a_{\mathrm{NS}}$. We also take for simplicity the gluon parameters to be the same as the singlet ones, i.e. $\beta_{g}=\beta_{q}$ and $a_{g}=a_{q}$, on the grounds that QCD evolution will anyway level small differences in the initial values of the various coefficients. More detailed fits could be obtained by relaxing these assumptions or increasing the order of the polynomial in $x$; however, our aim is not to propose a new parton parametrization, but rather to study the scale dependence of $g_{1}$, especially in the small $x$ region, where these details should make very little difference. The accuracy of present day data is in any case not good enough to justify fitting all these parameters independently [19].

Given that no clear-cut theoretical prediction for the small $x$ behaviour of $\Delta q$ and $\Delta g$ at a fixed scale exists, while we do not expect the present data to allow fitting the exponents $\alpha_{q}$ and $\alpha_{g}$, we will let them vary independently over the range of values $-1<\alpha_{q}, \alpha_{g} \leq 0$, and study the dependence of the results on this choice. The range of positive values

3 We will not consider logarithmic corrections to the power-like behaviour of the form (4.5) or (4.6) since the coefficient functions already display a logarithmic rise at small $x$, and thus it would be very hard to disentangle from the data such corrections to the boundary conditions. 
eq. (4.3) all give essentially the same behaviour of $g_{1}$ (because of the logarithmic rise in the coefficient function) and are thus all accounted for by the flat case $\alpha_{q}=0, \alpha_{g}=0$. The lower bound is necessary since the first moment of the parton distributions must be finite. The nonsinglet quark distribution, which does not mix with the gluon and with the sea, will be assumed to be valence-like, i.e. to have $\alpha_{\mathrm{NS}} \geq 0$; specifically we will take $\alpha_{\mathrm{NS}}=0.2$, which is the best-fit value of ref. [10] (where only the earlier data in the valence region were considered).

We are thus finally left with seven free parameters, namely the two parameters $\beta$ and $a$ controlling the large $x$ behaviour, the two small- $x$ exponents $\alpha_{q}$ and $\alpha_{g}$, and the three normalization coefficients $\eta_{q}, \eta_{\mathrm{NS}}$ and $\eta_{g}$. The normalization of the nonsinglet, however, is fixed by the fact that the first moment of $\Delta q^{\mathrm{NS}}$ is $Q^{2}$-independent to all orders because of the conservation of the nonsinglet axial current. It can thus be determined from the knowledge of the nonsinglet component of the matrix element of the axial current measured in hyperon $\beta$ decays:

$$
\eta_{\mathrm{NS}}=\int_{0}^{1} \Delta q^{\mathrm{NS}}\left(x, Q^{2}\right) d x=\frac{3}{4} g_{A}+\frac{1}{4} a_{8}
$$

where 28

$$
\begin{aligned}
& g_{A}=1.2573 \pm 0.0028 \\
& a_{8}=0.579 \pm 0.025 .
\end{aligned}
$$

The normalization of the gluon and singlet quark contributions to $g_{1}$ cannot be fixed analogously, due to the anomaly which afflicts the singlet axial curent. At a low scale, such as $Q^{2} \sim Q_{0}^{2}, \eta_{q}$ might be expected to be close to the normalization of the polarized quark distribution (i.e., the total fraction of spin carried by quarks) as calculated in quark models. One may estimate this from the normalization of the octet current $a_{8}$ eq. (4.7), which for valence quarks according to the Zweig rule will coincide with that of the singlet. The difference between $\eta_{q}$ and the observed small value of $\Gamma_{1}$ (which is much larger than the evolution effects) is then be made up by a large value of $\eta_{g}$ [5]. This interpretation of the data, however appealing, need not necessarily be true; we will therefore consider three different scenarios: i) a "maximal" gluon case, namely $\eta_{q}=a_{8}$; ii) the opposite limit where $\eta_{g}=0$ and any polarized gluons are generated dynamically; iii) a more general fit where both $\eta_{q}$ and $\eta_{g}$ are left as free parameters. Note that rather than using the experimental determinations of the first moment of $g_{1}$ as a constraint on $\eta_{q}$ and $\eta_{g}$, as in ref. [19], we will instead determine $\Gamma_{1}$ using our own fits to the $g_{1}$ data, evolved to a common scale. 
The results of the fit to the data are displayed in table 1 and figure 2 (in several typical cases) and can now be used to draw some quantitative conclusions on the scale dependence and small- $x$ behaviour of $g_{1}$. First, it is clear that a statistically significant evolution effect is seen in the data. Indeed, throughout the intermediate $x$ region $0.03 \leq x \leq 0.2$, where both experiments have data, there would appear to be a systematic discrepancy in that all of the E143 data [3] lie systematically about one standard deviation below the SMC data [2]. The difference is however seen to be entirely accounted for by the fact that in each $x$ bin the E143 data are taken at a smaller value of $Q^{2}$ than the SMC data. This is displayed explicitly in fig. 2a-c: in particular, fig. $2 \mathrm{c}$ shows that even if $\eta_{g}=0$ and $\alpha_{q}=0$, i.e. if the small $x$ behaviour of the initial conditions is very soft, the dynamically generated gluon polarization is sufficient to drive the required amount of perturbative evolution. Notice that (as shown explicitly by the calculation of ref. [10]) not enough evolution would be found if we had set $C_{g}=0$ in eq. (2.1). In fact, the total $\chi^{2}$ of the fit shown in fig. $2 \mathrm{c}$ (which is the least sensitive to this effect since it has minimal gluon) increases by more than three units if we set the coefficient functions to their one loop values eq. (2.5). This provides a posteriori evidence in favor of our approach, i.e. it supports the assumption that the inclusion of the next-to-leading order coupling of $\Delta g$ to $g_{1}$ even when the AltarelliParisi equations are only solved at leading order improves the determination of $g_{1}$; this can be thus interpreted, albeit with some caution, as direct experimental evidence for the anomaly-induced contribution of $\Delta g$ to $g_{1}$.

Even though the evolution of $\Gamma_{1}$ is fixed in a universal way by the anomalous dimension of the axial current $j_{5}^{\mu}$ (and is slight, since it starts at two loops), the evolution of $g_{1}$ for any finite value or range of $x$ is a leading order (potentially large) effect. However, it is not universal, but rather depends on the specific form of $\Delta q$ and $\Delta g$. In practice, the parameter which controls the exact amount of evolution is essentially the value of $\eta_{g}$, i.e., the size the gluon contribution at the starting scale. Large values of $\eta_{g}$ lead to significantly larger evolution. The value of $\eta_{g}$ cannot be determined from the data, but may be somewhat constrained. The limit on $\eta_{g}$ may be read off table 1 ; its actual value depends on the values of $\alpha_{q}$ and $\alpha_{g}$. In general, the data favor intermediate values of $\eta_{g}$, i.e. such that $\eta_{g}>0$, but still not large enough that the condition $\eta_{q}=a_{8}$ be satisfied at the starting scale.

On the contrary, no significant constraint is put by the data on the values of the small $x$ exponents $\alpha_{q}, \alpha_{g}$, as expected from fig. 1. Thus a good fit can be obtained with either "hard" and "soft" boundary conditions, such as, for example $\alpha_{q}=\alpha_{g}=-0.5$ and $\alpha_{q}=\alpha_{g}=0$ (see fig. 2a, b). In fact, with all combinations of small- $x$ exponents 
it is possible to accurately fit the data (table 1a) by suitably adjusting the value of $\eta_{g}$. However, for different classes of values of $\alpha_{q}$ and $\alpha_{g}$ the qualitative form of the perturbative evolution in the intermediate $x$ range $(0.03 \leq x \leq 0.1)$ may be significantly different. In particular, if $\alpha_{g}<\alpha_{q}$ (gluon more singular than quark) $g_{1}$ becomes rapidly large and negative at small $x$. It follows that only small values of $\eta_{g}$ (fig. 2 a) are allowed so that the decrease of $g_{1}$ falls outside the observed range. If instead $\alpha_{q} \leq \alpha_{g}$ (quark at least as singular as gluon), then the onset of the small- $x$ asymptotic behaviour (where $\Delta G$ and $\Delta q$ are anticorrelated, according to eq. (3.5)) tends to generate oscillatory interference patterns in $g_{1}$ (fig. $2 \mathrm{~d}$ ). These oscillations are more pronounced if $\eta_{g}$ is large (hence the evolution is stronger), and for soft boundary conditions (i.e. $\alpha_{q} \approx \alpha_{g} \gtrsim-0.3$ ), when the asymptotic double logarithmic growth eq. (3.7) only sets in slowly. This also allows us to rule out a maximal gluon for soft boundary conditions, since then fluctuations would be too large (fig. 2e). Finally, if $\alpha_{q}$ is very large, then it is possible to satisfy the condition $\eta_{q}=a_{8}$ even though in fact $\eta_{g}$ is very small (compatible with zero): in such case $g_{1}$ grows very large in the unobserved small- $x$ region. This scenario [30] is also compatible with the data (fig. 2f): an arbitrarily large contribution to $\Gamma_{1}$ concentrated at very small $x$ does not influence the evolution at larger $x$ and thus cannot be seen in $g_{1}(x)$ measured over any finite region of $x$.

Conversely, if $\eta_{g}$ is fixed (for example on the basis of theoretical prejudice), then the values of $\alpha_{q}, \alpha_{g}$ can be fitted (or at least constrained). In particular, if $\eta_{g}$ is very large (maximal), then only values $\alpha_{q}, \alpha_{g}<-0.5$, with $\alpha_{q}<\alpha_{g}$ are allowed, the corresponding minimum of the $\chi^{2}$ in the $\left(\alpha_{q}, \alpha_{g}\right)$ plane being rather narrow. If $\eta_{g}=0$, then a valence-like quark is favored $\left(\alpha_{q} \approx+0.5\right)$, albeit with a sizable uncertainty. For intermediate values of $\eta_{g}$ the $\chi^{2}$ is very smooth in the $\left(\alpha_{q}, \alpha_{g}\right)$ plane, and $\alpha_{q}$ and $\alpha_{g}$ are essentially unconstrained.

In order to check that these results are not a byproduct of our treatment of next-toleading corrections (which are included in the coefficient functions but not in the AltarelliParisi splitting functions), we have repeated our fits with the two alternative forms of gluon coefficient functions given in ref. [19]. The results do not change in a statistically significant way. 


\section{The First Moment}

On the basis of the quantitative knowledge on the evolution of $g_{1}$ acquired in the previous section, we may now discuss the values of its first moment $\Gamma_{1}$ eq. (1.1), and in particular the contribution to it from the small $x$ region

$$
\Gamma_{1}^{s x}\left(Q^{2}\right)=\int_{0}^{0.01} d x g_{1}\left(x, Q^{2}\right)
$$

Values of $\Gamma_{1}$ and $\Gamma_{1}^{s x}$ at various scales, and the value of the singlet contribution $a_{0}\left(Q^{2}\right)$ eq. (2.8) are given in table 2 , for several of the choices of the fit parameters which give good agreement with the data.

The contribution eq. (5.1) of the small $x$ extrapolation to $\Gamma_{1}$, at the scale of the data, turns out to be approximately the same for all reasonable fits (apart of course for the extremely singular case of fig. 2f). The uncertainty in the knowledge of the small- $x$ behaviour of the starting quark and gluon distributions eq. (4.1) is thus seen to have very little effect on the value of $\Gamma_{1}$.

However, there is a hitherto unnoticed sizable uncertainty in the value of $\Gamma_{1}$ at any given scale which is only indirectly related to the small $x$ extrapolation and is due to the scale dependence of $g_{1}$. This may be seen by comparing, for instance, the results of fitting the data with $\eta_{g}=0$ and $\alpha_{q}=0$ (fig. 2c), or with $\alpha_{q}=-0.5, \alpha_{g}=0$ and maximal gluon, $\eta_{q}=a_{8}$ (fig. $2 \mathrm{~d}$ ). These fits have comparable $\chi^{2}$, thus providing an equally accurate description of the data, and the same value of $\Gamma_{1}^{s x}\left(Q^{2}\right)$ at $Q^{2}=3 \mathrm{GeV}^{2}$. Nevertheless they lead to values of $\Gamma_{1}\left(Q^{2}\right)$ which differ by almost $10 \%$, hence to values of its singlet component which differ by almost a factor 2 .

The origin of this uncertainty can be understood by remembering that whereas the singlet contribution $a_{0}$ eq. (2.8) to $\Gamma_{1}$ is scale independent at leading order, the integral of $g_{1}$ restricted to a limited $x$ region is not. Inspection of the results of table 2 and fig. 2 shows that, for $\eta_{g}>0$, in the intermediate $x$ range $(0.03 \lesssim x \lesssim 0.2) g_{1}$, and thus the contribution to $\Gamma_{1}$ from this region, is a rather rapidly increasing function of $Q^{2}$; this is also manifestly displayed by the data. This implies that the contribution $\Gamma_{1}^{s x}$ from the (unmeasured) small $x$ region must decrease as $Q^{2}$ increases. However, this $Q^{2}$ dependence of $\Gamma_{1}^{s x}$ is extremely sensitive to the value of $\eta_{g}$ : if $\eta_{g}$ is large, then $\Gamma_{1}^{s x}$ decreases very rapidly, due to the large oscillations induced in $g_{1}$, while this is not the case if $\eta_{g}$ is small. But the value of $\eta_{g}$ is only weakly constrained by the data, since the corresponding evolution effects are mostly concentrated in the experimentally unaccessible small $x$ region. This implies that 
if $\eta_{g}$ is large, then $\Gamma_{1}$ can be substantially smaller at the starting scale because $g_{1}$ in the intermediate $x$ region, which provides the bulk of it, has been evolved more.

As a corollary to this result, we find that our determinations of $\Gamma_{1}$ are systematically smaller than those quoted in refs. [2,3]. This is because the data points which provide the bulk of $\Gamma_{1}$ are taken at a scale $Q^{2}$ larger than the nominal average scale of either experiment: for example in ref. [2] $Q_{\exp }^{2}(x)$ is always larger than $20 \mathrm{GeV}^{2}$ for $x$ above 0.1 , while $\left\langle Q^{2}\right\rangle=10 \mathrm{GeV}^{2}$ for this experiment. Since in this region $g_{1}$ increases as the scale increases the approximate treatment of the scale dependence in refs. [2,3] leads to an overestimate of $\Gamma_{1}$. 1 . Conversely, the small- $x$ extrapolation of ref. [2] overestimates the data, because it is obtained by extrapolating the smallest $x$ data points, which are taken at very low $Q^{2}\left(\sim 1 \mathrm{GeV}^{2}\right)$, and then assumed to apply at $<Q^{2}>=10 \mathrm{GeV}^{2}$; this turns out not to be the case for ref. [3] partly because their value of $<Q^{2}>=3 \mathrm{GeV}^{2}$ is closer to that of the small $x$ bins, and partly just because a straight line drawn from the smallest $x$ points of this experiment happens to be closer to our results.

This analysis is summarized pictorially in fig. 3, where we display the scale dependence of both $\Gamma_{1}$ and the small $x$ contribution to it, $\Gamma_{1}^{s x}$, for representative minimal, intermediate, and maximal values of $\eta_{g}$. Clearly, $\Gamma_{1}^{s x}$ decreases significantly as a function of scale if $\eta_{g}$ is maximal, whereas it is essentially scale-independent if it is minimal (the slight rise displayed in the figure in such case is not significant to the level of accuracy of the present treatment: it could be turned into a decrease for different forms of the next-to-leading corrections which we do not include). As a consequence, larger values of $\eta_{g}$ correspond to smaller values of $\Gamma_{1}$. Since however the value of $\eta_{g}$ cannot be fixed on the basis of theoretical arguments, and is not constrained by the data, this is to be considered as an intrinsic uncertainty on the determination of $\Gamma_{1}$. However, even with minimal values of $\eta_{g}$, inclusion of the next-to-leading evolution effects discussed here leads to a smaller value of $\Gamma_{1}$ than that found through the approximate treatment of the scale dependence given by the experimental collaborations [2, 3].

4 Notice however that part of the effect is due to the fact that our determination of $g_{1}$ from the data of ref. [3] is systematically smaller than that presented there, because of the kinematic higher twist effects included in their determination of $g_{1}$ from the measured asymmetry. 


\section{Discussion}

The main result of our analysis is that the next-to-leading order coupling of the polarized gluon distribution to $g_{1}$ has a sizable effect on the scale dependence of the structure function. This effect has important consequences for the extraction of the first moment $\Gamma_{1}$ of $g_{1}$ from the data because, even though $\Gamma_{1}$ is scale-independent at leading order, the contribution to it from any finite $x$ range (such as the experimentally accessible range) is not. In particular, the contribution to $\Gamma_{1}$ from the unobserved small- $x$ region turns out to be a decreasing function of the scale, this decrease being compensated by an approximately equal increase of the contribution from the measured region. The importance of the evolution effects is largely independent of the detailed shape of the quark and gluon distributions, but increases with the size of the polarized gluon distribution.

As a consequence, due to the neglect of these evolution effects, the value of $\Gamma_{1}$ is somewhat overestimated by present experiments, and the uncertainty, which is due to the lack of knowledge of the size of the polarized gluon, is substantially underestimated.

more accurate approximate $<Q^{2}>$ closer to the value of $Q_{\exp }^{2}(x)$ in the $x$ region gives the dominant contribution to $\Gamma_{1}$. Indeed, this considerably in reducing the uncertainty on the region. The largest uncertainty would then contribution which would be evolved uncertainty in the knowledge of the small $x$ behaviour of polarized parton distributions leads to a comparatively much smaller ambiguity, at least excluding the possibility of a very large contribution to $g_{1}$ from the very small $x$ region (such as a delta-like contribution). A more accurate determination of $\Gamma_{1}$ and especially of the associated error can only be obtained by including the full scale dependence in the analysis of the data. From table 2 we suggest the value

$$
\Gamma_{1}\left(Q^{2}=3 \mathrm{GeV}^{2}\right)=0.120 \pm 0.005 \pm 0.005
$$

where the first error is statistical and the second is an optimistic estimate of the theoretical uncertainty in the scale dependence due to the uncertainty in the size of the gluon distribution. The systematic error, which is typically of order 0.010 , must still be added.

The intrinsic ambiguity related to evolution could be substantially reduced if more precise data in the intermediate $x$ region $(0.03 \lesssim x \lessgtr 0.2)$ were available for somewhat different values of $Q^{2}$ : because of the instability of $g_{1}$ under perturbative evolution even a moderate improvement in experimental knowledge could result in much more stringent theoretical constraints on the size of the polarized gluon distribution, which in turn would 
be enough to determine the small $x$ behaviour of $g_{1}$ to much better accuracy. Such information would thus be more conclusive than direct information on $g_{1}$ in the small $x$ region itself. The determination even of the qualitative behaviour of $g_{1}$ at small $x$, in the same way as in the unpolarized case [8] would instead only be feasible with a kinematic coverage of the small $x$ region comparable to that which HERA data provide at present for $F_{2}$.

In short, the scale dependence of $g_{1}$ induced by the axial anomaly turns out to be an essential ingredient in its phenomenology: a more detailed understanding of it will only be possible once the actual size of the gluon polarization is known more accurately. On the theoretical side, the determination of the full set of two loop Altarelli-Parisi splitting function will allow a fully consistent treatment. It will then perhaps be possible to extract information on the polarized gluon distribution, which is of considerable intrinsic theoretical interest, directly from structure function data even over a moderate range of $x$ and $Q^{2}$.

Acknowledgement: We thank G. Altarelli for discussions, and P. Nason and S. Rollet for useful suggestions. 


\begin{tabular}{|c|c|c|c|c|c|c|}
\hline$\alpha_{q}$ & $\alpha_{g}$ & $\beta$ & $a$ & $\eta_{q}$ & $\eta_{g}$ & $\chi^{2}$ \\
\hline \multicolumn{7}{|c|}{ Fitted gluon: } \\
\hline 0.0 & 0.0 & $2.95 \pm 0.50$ & $1.44 \pm 1.93$ & $0.18 \pm 0.88$ & $0.22 \pm 0.50$ & 31.0 \\
\hline 0.0 & -0.2 & $2.94 \pm 0.47$ & $1.43 \pm 1.74$ & $0.18 \pm 0.72$ & $0.22 \pm 0.42$ & $\left.30.9^{\mathrm{b}}\right)$ \\
\hline 0.0 & -0.5 & $2.98 \pm 0.49$ & $1.62 \pm 1.96$ & $0.16 \pm 0.73$ & $0.18 \pm 0.51$ & 30.9 \\
\hline 0.0 & -0.9 & $3.07 \pm 0.38$ & $2.11 \pm 1.59$ & $0.15 \pm 0.45$ & $0.09 \pm 1.58$ & 31.1 \\
\hline-0.2 & 0.0 & $2.96 \pm 0.49$ & $1.57 \pm 1.97$ & $0.21 \pm 0.11$ & $0.35 \pm 0.59$ & 31.0 \\
\hline-0.5 & 0.0 & $2.99 \pm 0.48$ & $1.78 \pm 2.12$ & $0.32 \pm 0.19$ & $0.74 \pm 0.86$ & 31.0 \\
\hline-0.9 & 0.0 & $3.45 \pm 0.26$ & $5.83 \pm 2.36$ & $0.43 \pm 1.00$ & $0.00 \pm 1.12$ & 33.0 \\
\hline-0.2 & -0.2 & $2.94 \pm 0.46$ & $1.54 \pm 1.76$ & $0.20 \pm 0.87$ & $0.35 \pm 0.48$ & 30.8 \\
\hline-0.5 & -0.5 & $2.98 \pm 0.40$ & $1.91 \pm 1.64$ & $0.28 \pm 0.10$ & $0.63 \pm 0.54$ & $30.4^{\mathrm{a})}$ \\
\hline-0.9 & -0.9 & $3.12 \pm 0.33$ & $3.00 \pm 1.70$ & $0.97 \pm 0.33$ & $2.80 \pm 1.58$ & 29.8 \\
\hline \multicolumn{7}{|c|}{ Maximal gluon: } \\
\hline 0.0 & 0.0 & $2.17 \pm 0.34$ & $-0.61 \pm 0.30$ & 0.58 & $2.02 \pm 0.17$ & 72.0 \\
\hline 0.0 & -0.2 & $2.11 \pm 0.34$ & $-0.62 \pm 0.31$ & 0.58 & $1.96 \pm 0.18$ & $83.2^{\mathrm{e})}$ \\
\hline 0.0 & -0.5 & $1.98 \pm 0.35$ & $-0.62 \pm 0.32$ & 0.58 & $1.99 \pm 0.20$ & 104.7 \\
\hline 0.0 & -0.9 & $1.76 \pm 0.42$ & $-0.66 \pm 0.37$ & 0.58 & $4.48 \pm 0.57$ & 141.5 \\
\hline-0.2 & 0.0 & $2.08 \pm 0.42$ & $-0.61 \pm 0.39$ & 0.58 & $2.08 \pm 0.17$ & 51.1 \\
\hline-0.5 & 0.0 & $2.41 \pm 0.53$ & $0.02 \pm 0.86$ & 0.58 & $1.87 \pm 0.18$ & $33.2^{\mathrm{d})}$ \\
\hline-0.9 & 0.0 & $3.42 \pm 0.26$ & $5.30 \pm 2.10$ & 0.58 & $0.24 \pm 0.17$ & $33.3^{\mathrm{f})}$ \\
\hline-0.2 & -0.2 & $2.02 \pm 0.42$ & $-0.61 \pm 0.39$ & 0.58 & $2.04 \pm 0.18$ & 59.2 \\
\hline-0.5 & -0.5 & $2.17 \pm 0.61$ & $-0.20 \pm 0.85$ & 0.58 & $2.05 \pm 0.20$ & 40.5 \\
\hline-0.9 & -0.9 & $3.36 \pm 0.26$ & $4.56 \pm 1.95$ & 0.58 & $0.98 \pm 0.51$ & 31.2 \\
\hline \multicolumn{7}{|c|}{ Minimal gluon: } \\
\hline 0.0 & - & $3.09 \pm 0.33$ & $2.23 \pm 1.29$ & $0.14 \pm 0.33$ & 0 & $31.1^{\mathrm{c})}$ \\
\hline-0.2 & - & $3.15 \pm 0.31$ & $2.72 \pm 1.39$ & $0.15 \pm 0.34$ & 0 & 31.3 \\
\hline-0.5 & - & $3.26 \pm 0.29$ & $3.76 \pm 1.64$ & $0.17 \pm 0.39$ & 0 & 31.7 \\
\hline-0.9 & - & $3.45 \pm 0.26$ & $5.83 \pm 2.36$ & $0.43 \pm 1.00$ & 0 & 33.0 \\
\hline
\end{tabular}

Table 1: Best-fit parameters and $\chi^{2}$ for the fit of $g_{1}$ to the data of refs. [2],3]: the letters a)-f) refer to the plots in fig. 2 . 


\begin{tabular}{|l|c|c|c|c|c|c|c|}
\hline & $\Gamma_{1}(1)$ & $\Gamma_{1}(3)$ & $\Gamma_{1}(10)$ & $\Gamma_{1}^{\mathrm{sx}}(1)$ & $\Gamma_{1}^{\mathrm{sx}}(3)$ & $\Gamma_{1}^{\mathrm{sx}}(10)$ & $a_{0}(3)$ \\
\hline \multicolumn{7}{|c|}{ Fitted gluon: } \\
\hline a) & $0.108 \pm 0.006$ & $0.118 \pm 0.006$ & $0.123 \pm 0.006$ & 0.007 & 0.003 & 0.001 & $0.102 \pm 0.060$ \\
b) & $0.110 \pm 0.005$ & $0.119 \pm 0.005$ & $0.123 \pm 0.005$ & 0.005 & 0.004 & 0.004 & $0.111 \pm 0.054$ \\
\hline \multicolumn{7}{|l|}{ Maximal gluon: } \\
\hline d) & $0.101 \pm 0.005$ & $0.116 \pm 0.005$ & $0.122 \pm 0.005$ & 0.015 & 0.005 & -0.004 & $0.074 \pm 0.051$ \\
f) & $0.147 \pm 0.005$ & $0.158 \pm 0.005$ & $0.162 \pm 0.005$ & 0.041 & 0.042 & 0.042 & $0.496 \pm 0.050$ \\
\hline \multicolumn{2}{|l|}{ Minimal gluon: } \\
\hline c) & $0.113 \pm 0.003$ & $0.122 \pm 0.003$ & $0.125 \pm 0.003$ & 0.005 & 0.006 & 0.007 & $0.136 \pm 0.031$ \\
\hline
\end{tabular}

Table 2: Values of the first moment $\Gamma_{1}\left(Q^{2}\right)$ eq. (1.1), at $Q^{2}=1,3,10 \mathrm{GeV}^{2}$, its small $x$ component eq. (5.1), and the matrix element $a_{0}$ of the singlet axial current for several typical fits from table 1: the letters a)-f) refer to the plots in fig. 2 . 


\section{References}

[1] For recent reviews see G. Altarelli and G. Ridolfi, Nucl. Phys. B (Proc. Suppl.) 39B (1995) 106;

S. Forte, preprint CERN-TH.7453/94.

[2] SMC Collaboration, D. Adams et al., Phys. Lett. B329 (1994) 399.

[3] E143 Collaboration, K. Abe et al., Phys. Rev. Lett. 74 (1995) 346.

[4] E143 Collaboration, R. Arnold et al., preliminary results presented at ICHEP94, Glasgow, August 1994.

[5] G. Altarelli and G. G. Ross, Phys. Lett. B212 (1988) 391.

[6] M. A. Ahmed and G. G. Ross, Phys. Lett. B56 (1975) 385.

[7] A. De Rujula, S.L. Glashow, H.D. Politzer, S.B. Treiman, F. Wilczek and A. Zee, Phys. Rev. D10 (1974) 1649.

[8] R. D. Ball and S. Forte, Phys. Lett. B335 (1994) 77; Phys. Lett. B336 (1994) 77.

[9] J. Ellis and M. Karliner, Phys. Lett. B313 (1993) 131.

[10] G. Altarelli, P. Nason and G. Ridolfi, Phys. Lett. B320 (1994) 152.

[11] E.B. Zijlstra and W.L. van Neerven, Nucl. Phys. B417 (1994) 61.

[12] G. Altarelli, Phys. Rep. 81 (1982) 1.

[13] G. Altarelli and G. Parisi, Nucl. Phys. B126 (1977) 298.

[14] See e.g. R. Jackiw in S. Treiman, R. Jackiw, B. Zumino and E. Witten, Current Algebra and Anomalies (World Scientific, Singapore, 1985).

[15] R. D. Carlitz, J. C. Collins and A. H. Mueller, Phys. Lett. B214 (1988) 229.

[16] G. Altarelli and B. Lampe, Zeit. Phys. C47 (1990) 315;

W. Vogelsang, Zeit. Phys. C50 (1991) 275.

[17] S. Forte, Nucl. Phys. B331 (1990) 1.

[18] G. Altarelli and W.J. Stirling, Particle World 1 (1989) 40.

[19] T. Gehrmann and W.J. Stirling, Durham preprint DTP/94/38 (1994).

[20] J. Ellis and M. Karliner, Phys. Lett. B341 (1995) 397

[21] M. B. Einhorn and J. Soffer, Nucl. Phys. B74 (1986) 714.

[22] NMC Collaboration, P. Amaudruz et al., Phys. Lett. B295 (1992) 159.

[23] L. W. Whitlow et al., Phys. Lett. B250 (1990) 193.

[24] See S. J. Brodsky, M. Burkardt and I. Schmidt, preprint SLAC-PUB-6087 (1994) and ref. therein

[25] R. L. Heimann, Nucl. Phys. B64 (1973) 429.

[26] See e.g. B. Badełek et al., Rev. Mod. Phys. 64 (1992) 927.

[27] S. D. Bass and P. V. Landshoff, Phys. Lett. B336 (1994) 537.

[28] F. E. Close and R. G. Roberts, Phys. Lett. B336 (1994) 257.

[29] M. Virchaux and A. Milsztajn, Phys. Lett. B274 (1992) 221.

[30] F. E. Close and R. G. Roberts, Phys. Rev. Lett. 60 (1988) 1471. 


\section{Figure Captions}

Fig. 1 Contour plot of $\ln g_{1}$ computed from the small $x$ evolution equation eq. (3.2) in the plane of the variables defined in eq. (3.3), with $Q_{0}=1 \mathrm{GeV}$ and $x_{0}=0.1$. The polarized gluon and singlet quark distributions are evolved up from the form eq. (4.1) with $\beta=3, \eta_{q}=0.6, \eta_{g}=2.5$ (maximal gluon), $a=0$ and a) $\alpha_{q}=0, \alpha_{g}=-0.2$ (soft); b) $\alpha_{q}=-0.5, \alpha_{g}=-0.5$ (hard). A scatter plot of the SMC $(+)$ and E143 $(\times)$ data is superposed for reference.

Fig. 2 Evolution of the structure function $g_{1}$ compared to the SMC (crosses) and E143 (diamonds) data. The full set of parameters for the various fits are given in table 1. a) fitted $\eta_{g}$, hard boundary conditions; b) fitted $\eta_{g}$, soft boundary conditions; c) dynamically generated gluon $\left(\eta_{g}=0\right)$; d) maximal gluon, hard boundary conditions; e) maximal gluon, soft boundary conditions; f) Ellis-Jaffe quark with small gluon contribution.

Fig. 3 Scale dependence of $\Gamma_{1}$ eq. (1.1) (a) and the the small $x(x \leq 0.01)$ contribution to it eq. (5.1) (b). Solid line: fitted gluon (as in fig. 2a); dot-dashed line: dynamically generated gluon (as in fig. 2c); dashed line: maximal gluon (as in fig. 2d). 


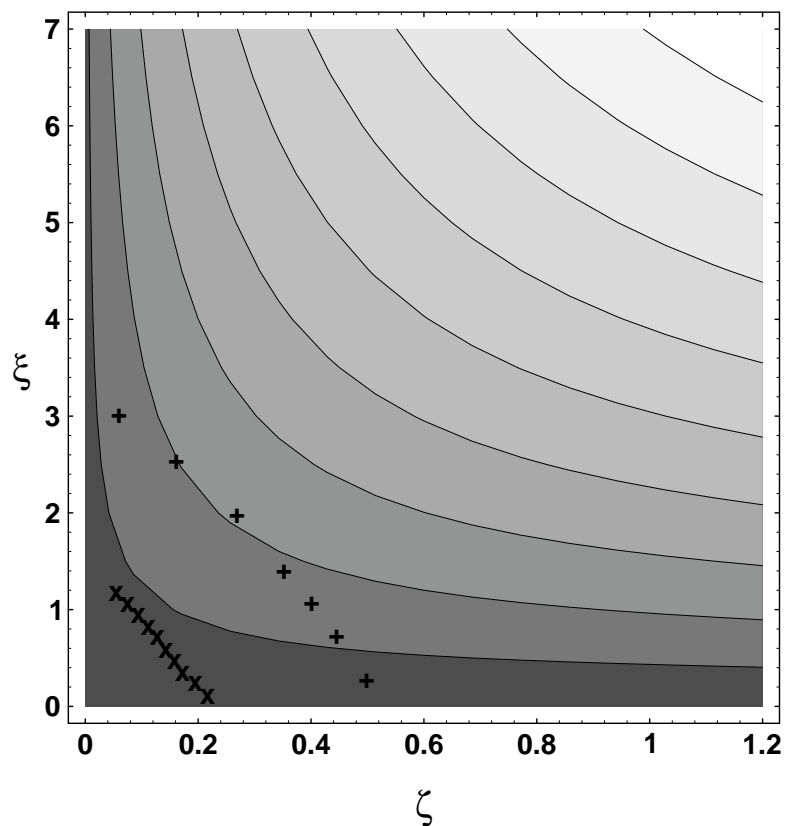

Fig. 1a

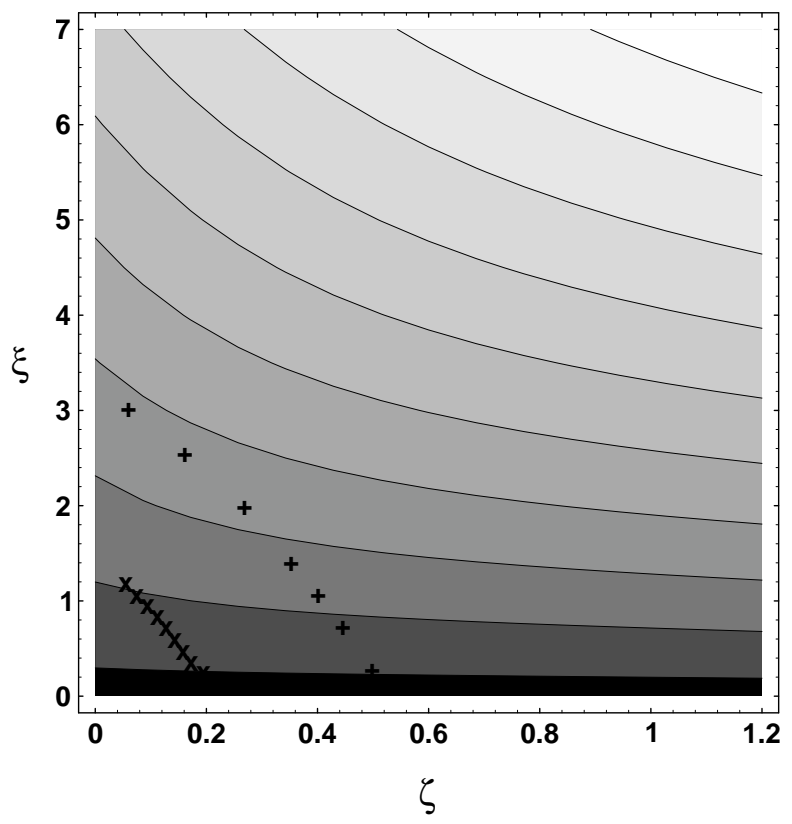

Fig. 1b 


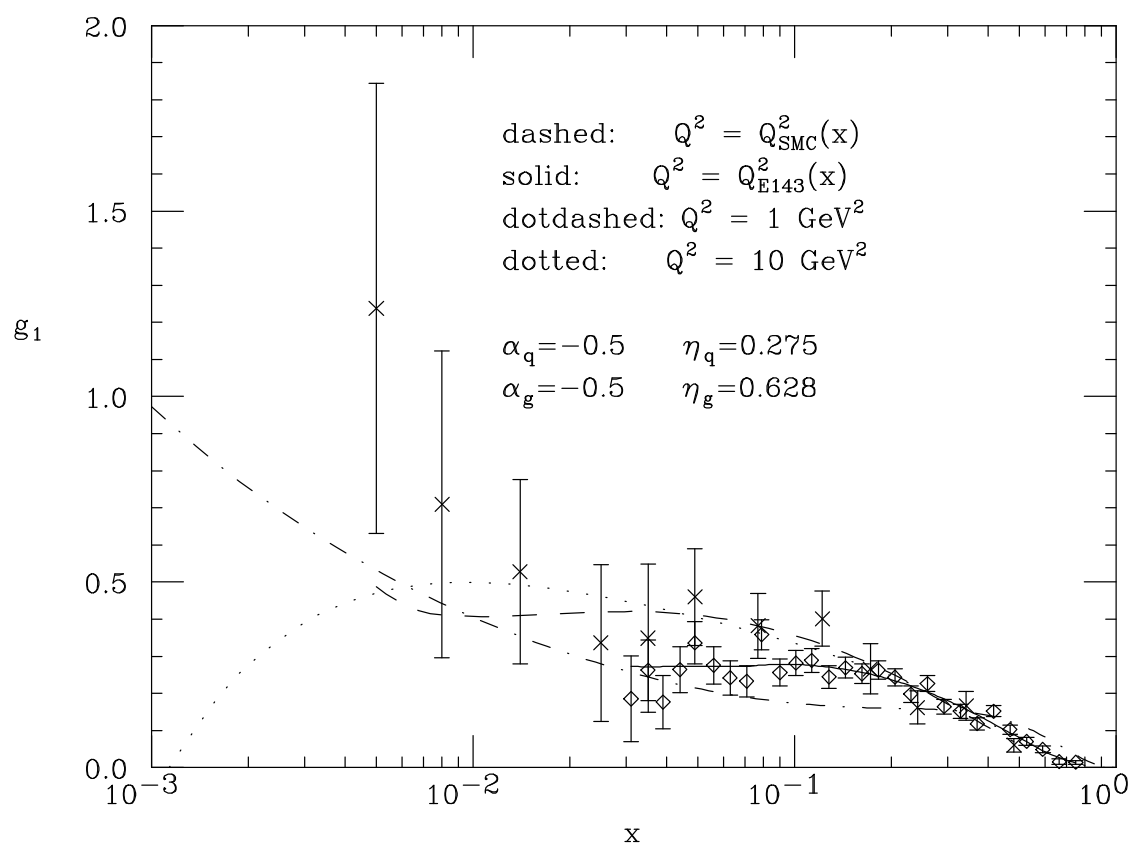

Fig. 2a

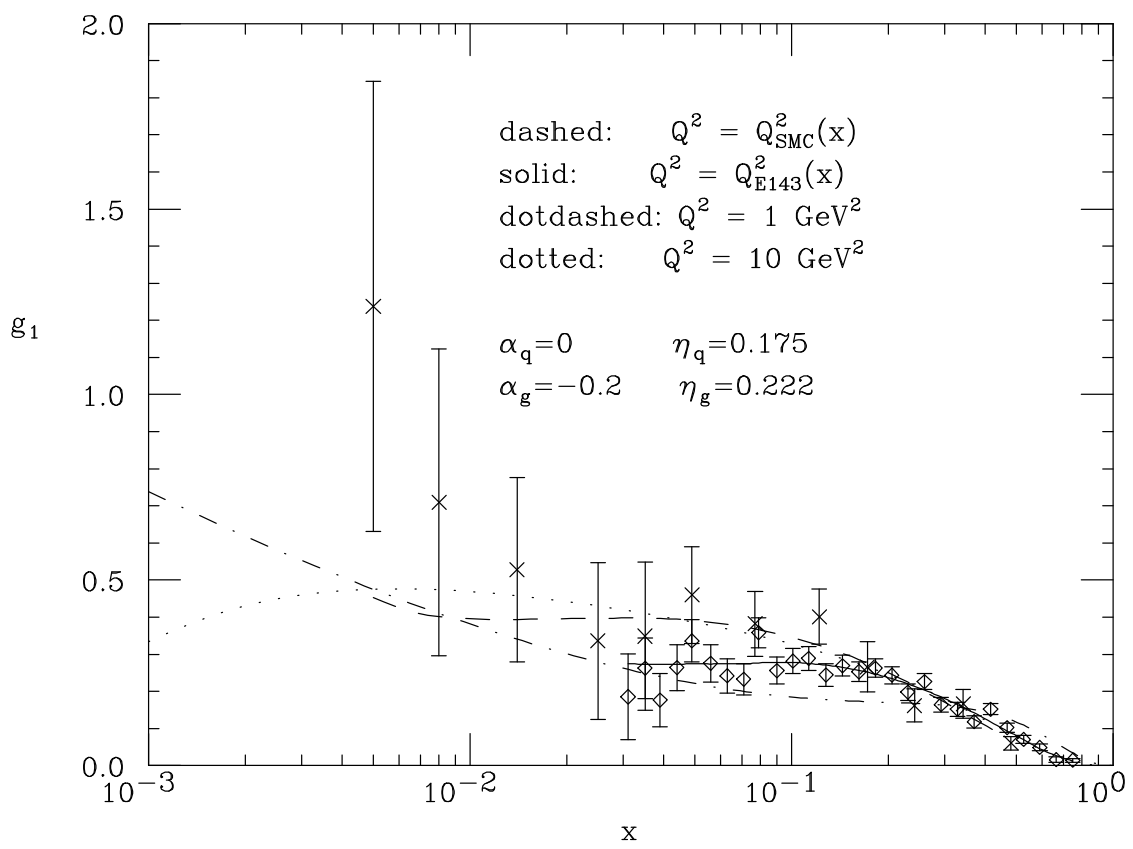

Fig. 2b 


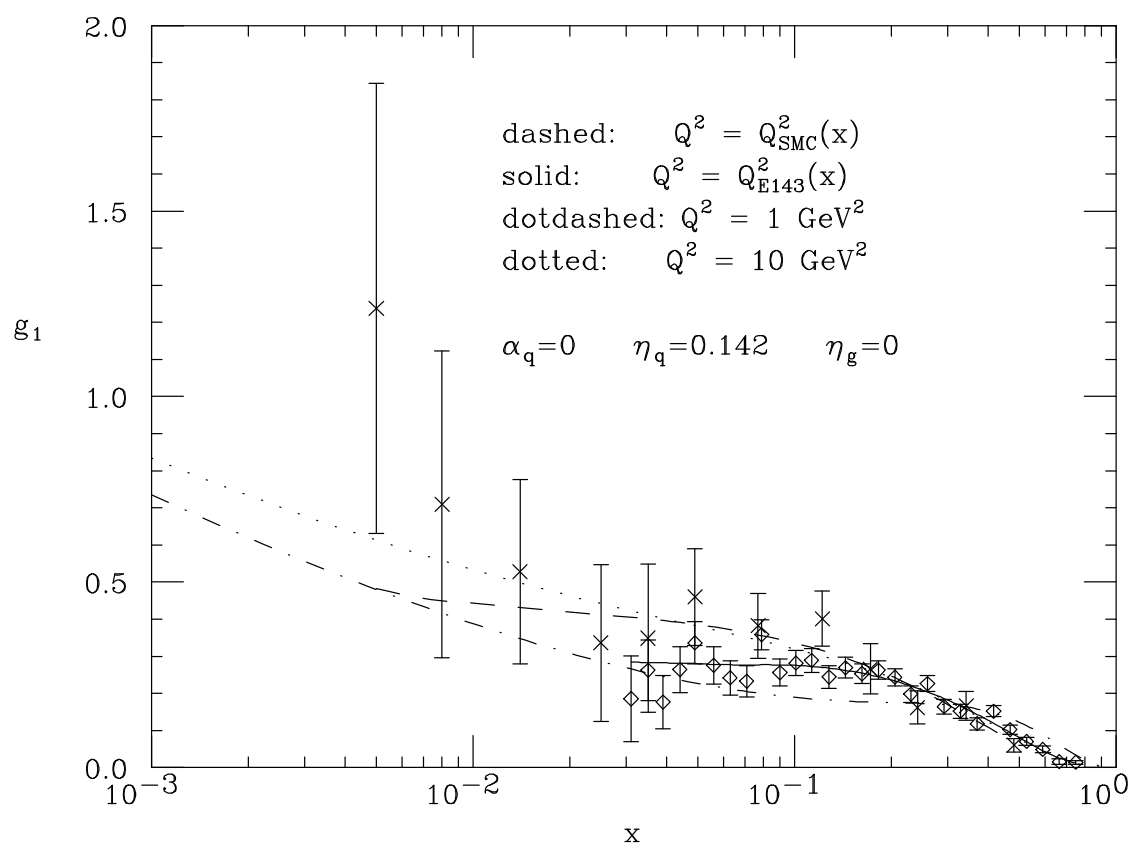

Fig. 2c

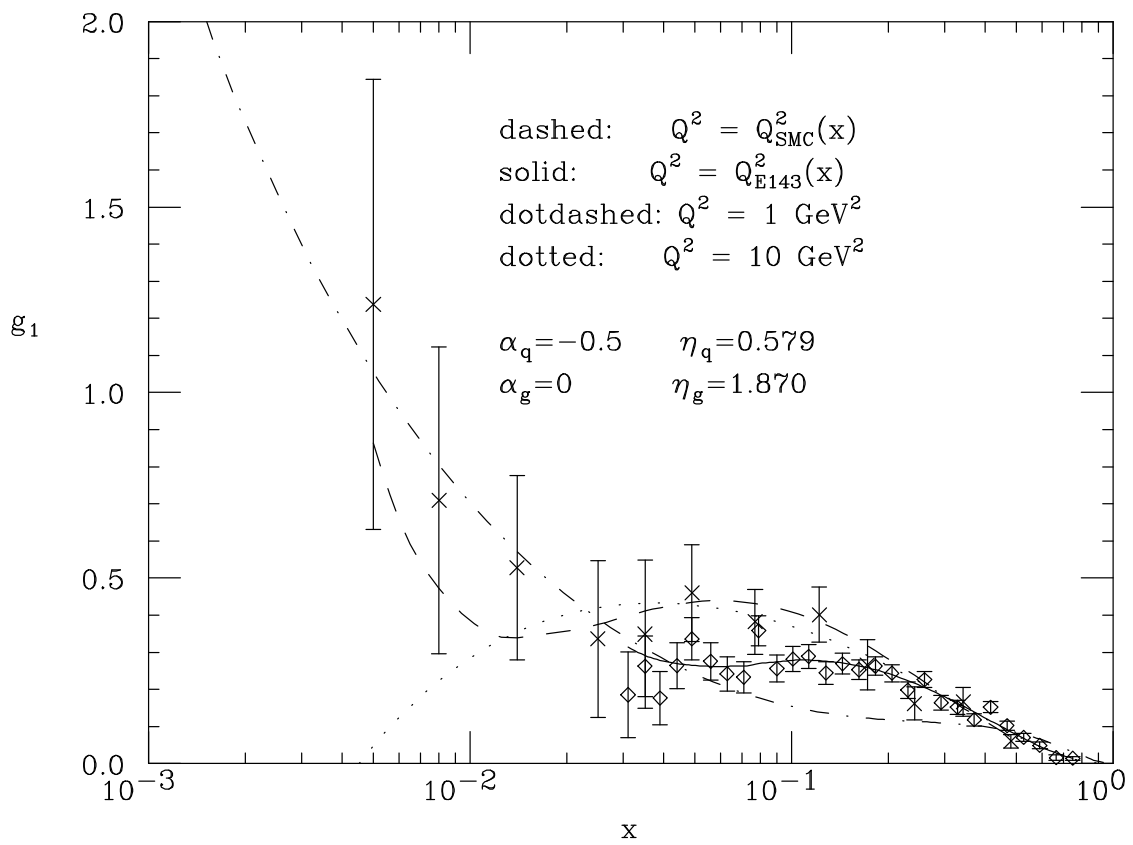

Fig. 2d 


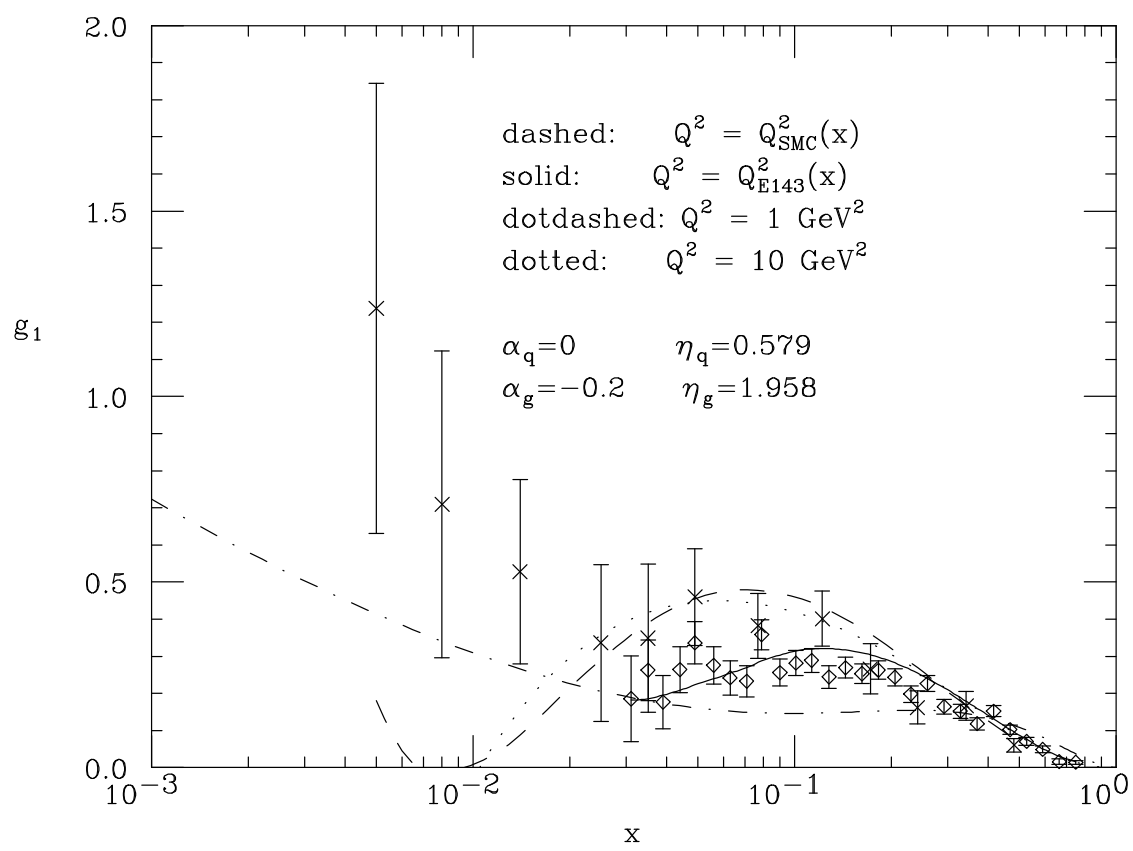

Fig. 2e

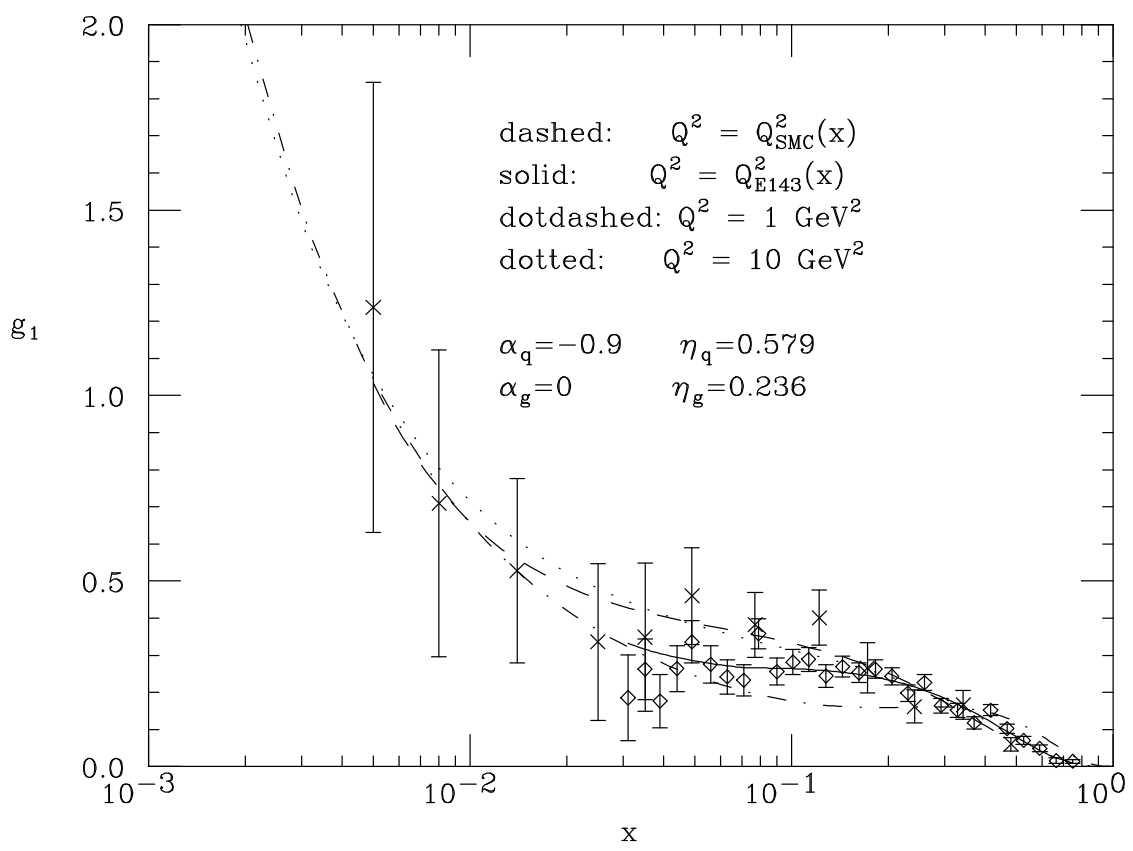

Fig. 2f 


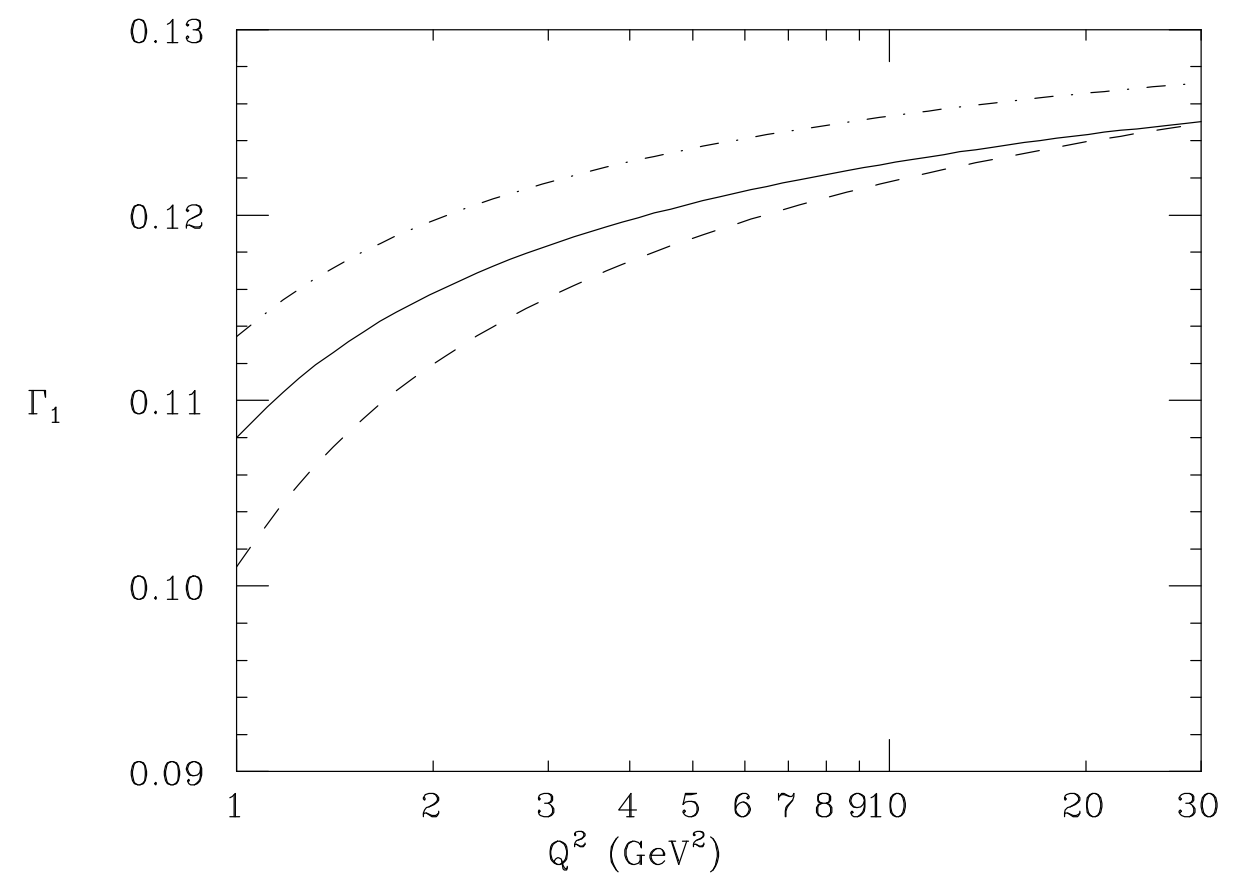

Fig. 3a

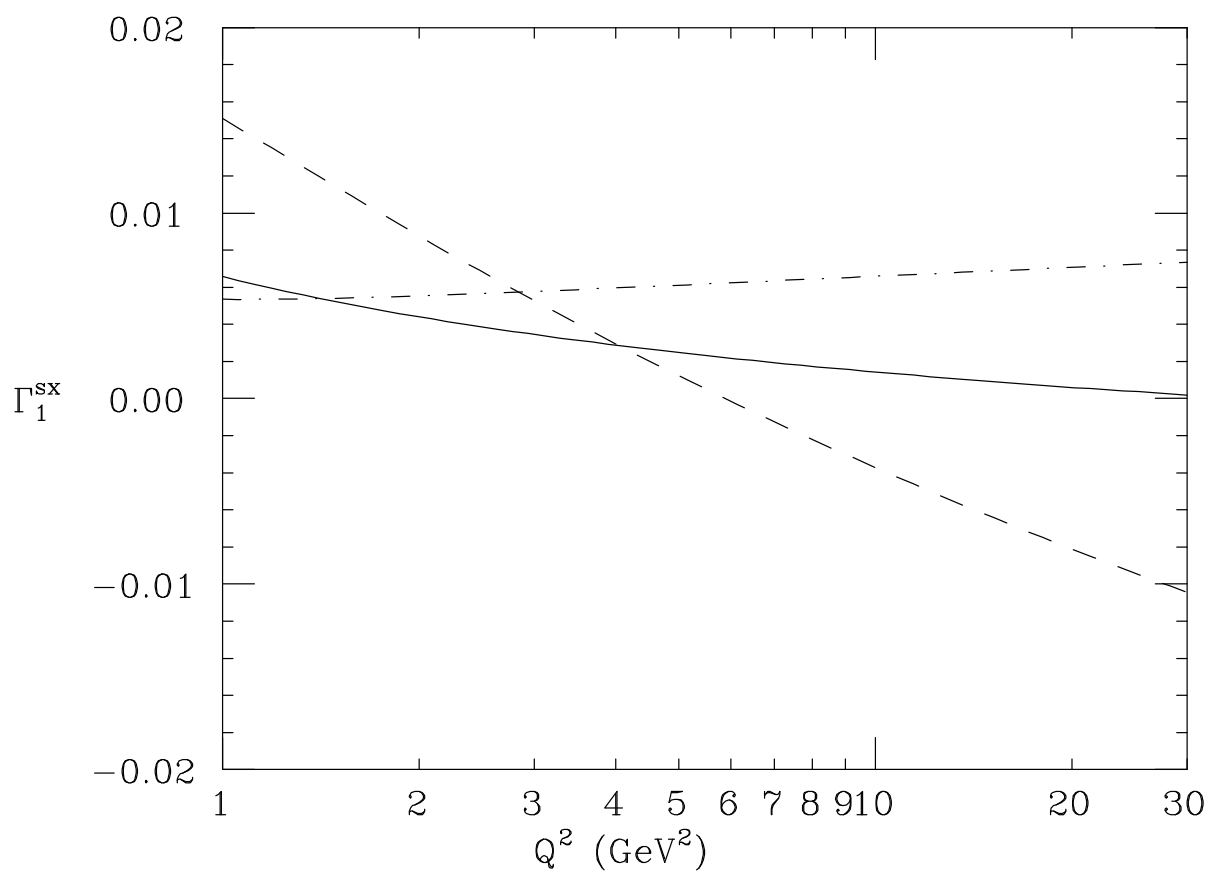

Fig. 3b 